Pacific Journal of Mathematics

SECTIONS AND SUBSETS OF SIMPLEXES 


\title{
SECTIONS AND SUBSETS OF SIMPLEXES
}

\author{
ALDo J. LAzAR
}

There is a locally convex space $E$ and a compact simplex $S \subset E$ with the following property: for any metrizable compact convex subset $K$ of a locally convex space there is a subspace $M \subseteq E$ such that $K$ is affinely homeomorphic to $M \cap S$. One possible choice is $E=l_{1}$ with the $w^{*}$ topology induced by $c$ and

$$
S=\left\{\left(a_{n}\right): \sum_{n=1}^{n} a_{n}=1, a_{n} \geqq 0, n=1,2, \cdots\right\} .
$$

If $X$ is a Banach space and $S \subset X$ is a compact simplex, then for each $\varepsilon>0$ there is an operator $T: X \rightarrow X$ with finite dimensional range such that $\|T(x)-x\|<\varepsilon$ for all $x \in S$. Every infinite dimensional Banach space $X$ contains a compact set $K$ for which there is no bounded simplex $S \subset X$ with $K \subset S$.

In a recent paper Phelps [12] gave an example of a three dimensional section of a compact simplex which is not a convex polytope. Our first result shows that this is not an exception: each metrizable compact convex subset of a locally convex space can be represented as the intersection of a certain compact simplex with a suitable linear space. In $\S 3$ we pass from sections of simplexes to compact simplexes in Banach spaces. The interest in their investigation is motivated by the fact that the identity operator of a Banach space can be approximated on a compact simplex of the space by operators having finite dimensional ranges (Theorem 3). Obviously the same property is shared by any set contained in a compact simplex of a Banach space. One says that a Banach space $X$ has the approximation property [6, p. 165] if for every compact $K \subset X$ and every $\varepsilon>0$ there is a bounded linear operator $T: X \rightarrow X$ with finite dimensional range such that $\|T(x)-x\|<\varepsilon$ for any $x \in K$. It is an open problem if every Banach space has the approximation property. Theorem 3 states that such an operator $T$ can always be found when $K$ is a subset of a compact simplex $S \subseteq X$. However our result is probably very far from leading to an affirmative solution of this problem of Grothendieck. Indeed, in $\S 4$ we prove that every infinite dimensional Banach space contains a compact set which is too large to be included in a bounded simplex of the space (Corollary 6). At the end we mention some open problems related to the material contained in this paper.

1. We shall consider only linear spaces over the real field $R$. 
Let $S$ be a convex subset of a linear space $E$. Passing to $E \times R$ if necessary, we may suppose that $S$ is situated in a hyperplane of $E$ which misses the origin. $S$ is called a simplex if the cone $C$ with the vertex at the origin generated by $S$ induces a lattice order in $C-C$.

For a compact convex subset $K$ of a locally convex linear space we denote by $A(K)$ the Banach space of all continuous affine functionals on $K$ endowed with the supremum norm. $A(K)$ has an order structure too determined by the cone of the nonnegative functions. $A^{*}(K)$ is an ordered Banach space with the dual order to that in $A(K)$. For $k \in K$ define $k^{*} \in A^{*}(K)$ by $k^{*}(f)=f(k), f \in A(K)$. The map $k \rightarrow k^{*}$ is an affine homeomorphism of $K$ onto the positive face of the unit ball in $A^{*}(K)$. If $f^{*} \in A^{*}(K), f^{*} \geqq 0$ and $f^{*}\left(1_{K}\right)=1$ (we denote by $1_{K}$ the function identically equal to 1 on $K$ ), then $f^{*}=k^{*}$ for a certain $k \in K$. The linear span of $\left\{k^{*}: k \in K\right\}$ is the entire space $A^{*}(K)$. If $K$ is a compact simplex then $A^{*}(K)$ is an $L$ space (cf. [14]).

The space $c$ consists of all the convergent real sequences $x=$ $\left(x_{1}, x_{2}, \cdots, x_{n}, \cdots\right)$ with $\|x\|=\sup \left|x_{n}\right|$. Let $\Gamma$ be a set. Then $l_{1}(\Gamma)$ denotes the space of all real functions $x=(x(\gamma))_{\gamma \in \Gamma}$ on $\Gamma$ which satisfy $\sum_{\gamma \in \Gamma}|x(\gamma)|<\infty$; the norm is $\|x\|=\sum_{\gamma \in \Gamma}|x(\gamma)|$. The space of all bounded real functions on $\Gamma$ with the supremum norm is denoted $l_{\infty}(\Gamma)$; one has $l_{1}^{*}(\Gamma)=l_{\infty}(\Gamma)$. If $\Gamma$ is countable we denote $l_{1}(\Gamma)$ by $l_{1}$. The dual of $c$ is isometrically isomorphic to $l_{1}$.

2. For the following theorem $S$ will denote the positive face of the unit ball in $l_{1}$, i.e.,

$$
S=\left\{\left(y_{n}\right): \sum_{n=1}^{\infty} y_{n}=1, y_{n} \geqq 0, n=1,2, \cdots\right\} .
$$

Obviously $S$ is a simplex. With the $w^{*}$-topology induced by $c$ in $l_{1} S$ is compact.

Theorem 1. Consider $l_{1}=c^{*}$ with the $w^{*}$-topology. For any metrizable compact convex subset $K$ of a locally convex space there is a subspace $M \subset l_{1}$ such that $M \cap S$ is affinely homeomorphic to $K$.

Proof. Let $\left\{f_{n}\right\}_{n=1}^{\infty}$ be a norm dense sequence in the positive cone of $A(K)$ such that $f_{n} \neq 0_{K}, n=1,2, \ldots$. Denote

$$
g_{n}=2^{-n}\left\|f_{n}\right\|^{-1}, n=1,2, \cdots \text { and } g_{0}=1_{K}-\sum_{n=1}^{\infty} g_{n} .
$$

Define the operator $T: c \rightarrow A(K)$ as follows: if $x=\left(x_{0}, x_{1}, \cdots, x_{n}, \cdots\right) \in c$ then $T(x)=\sum_{n=0}^{\infty} x_{n} g_{n}$. We shall show that $M=T^{*}\left(A^{*}(K)\right) \subset l_{1}$ has 
the desired properties. Clearly it is enough to prove that $T^{*}$ is one to one and that $T^{*}$ maps $\left\{k^{*}: k \in K\right\}$ onto $M \cap S$.

The first assertion about $T^{*}$ is a consequence of the fact that $T(c)$ is dense in $A(K)$. We prove now the second claim. $T$ is a positive map of $c$ into $A(K)$ hence $T^{*}\left(k^{*}\right)$ is nonnegative for every $k \in K$. Put $e=(1,1, \cdots) \in c$. Then we have

$$
T^{*}\left(k^{*}\right)(e)=k^{*}(T(e))=k^{*}\left(1_{K}\right)=1,
$$

which implies that $T^{*}\left(k^{*}\right) \in S$. Assume now that $T^{*}\left(f^{*}\right) \in S$ for $f^{*} \in A^{*}(K)$. Then

$$
f^{*}\left(g_{n}\right)=f^{*}\left(T\left(e_{n}\right)\right)=T^{*}\left(f^{*}\right)\left(e_{n}\right) \geqq 0, n=1,2 \cdots
$$

where $e_{n}$ is the $n$-th unit vector in $c$. By the choice of the functions $f_{n}$ we conclude that $f^{*} \geqq 0$. Moreover, since

$$
f^{*}\left(1_{K}\right)=f^{*}(T(e))=T^{*}\left(f^{*}\right)(e)=1,
$$

there is $k \in K$ such that $f^{*}=k^{*}$.

REMARKs. If $K$ is a finite dimensional set then the subspace $M$ of $l_{1}$ given by the preceding proof is finite dimensional hence $w^{*}$-closed in $l_{1}$. However in general $M$ is not even norm closed. Indeed, if $K$ is the set of all probability measures in $C^{*}(0,1)$ with the $w^{*}$-topology then $A^{*}(K)$ is not separable and by Banach's open mapping theorem it cannot be mapped by a one-to-one bounded linear operator onto a norm closed subspace of $l_{1}$. We do not know whether for any metrizable compact convex $K$ one can find a norm closed subspace $M$ of $l_{1}$ such that $K$ is affinely homeomorphic to $S \cap M$.

The metrizability condition imposed on $K$ is essential for the existence of an embedding into a compact simplex. There is a nonmetrizable compact convex set which is not affinely homeomorphic to any subset of a compact simplex.

Let $\Gamma$ be an uncountable set. It follows from [9, Th. 4.7] that there is a compact convex subset $K$ of a locally convex space such that $l_{1}(\Gamma)=A(K)$. There is no compact simplex which contains a subset affinely homeomorphic to $K$. Assume the contrary and let $\alpha$ be an affine homeomorphism of $K$ into the compact simplex $S$. Define $T: A(S) \rightarrow A(K)$ by $T(f)(k)=f(\alpha(k)), f \in A(S), k \in K$. The adjoint map $T^{*}: A^{*}(K) \rightarrow A^{*}(S)$ is an extension of $\alpha$. Moreover, $T^{*}$ is one to one. Indeed, let $k_{i}, k_{i}^{\prime} \in K$ and $\lambda_{i}, \lambda_{i}^{\prime} \geqq 0, i=1,2$ with

$$
T^{*}\left(\lambda_{1} k_{1}^{*}-\lambda_{2} k_{2}^{*}\right)=T^{*}\left(\lambda_{1}^{\prime} k_{1}^{\prime *}-\lambda_{2}^{\prime} k_{2}^{\prime *}\right) \text {. }
$$

Then

$$
\lambda_{1} \alpha\left(k_{1}\right)+\lambda_{2}^{\prime} \alpha\left(k_{2}^{\prime}\right)=\lambda_{1}^{\prime} \alpha\left(k^{\prime}\right)+\lambda_{2} \alpha\left(k_{2}\right)
$$


and by the well known property of the $L$-norm, $\lambda_{1}+\lambda_{2}^{\prime}=\lambda_{1}^{\prime}+\lambda_{2}$. It follows then easily from (1) that $\lambda_{1} k_{1}+\lambda_{2}^{\prime} k_{2}^{\prime}=\lambda_{1}^{\prime} k_{1}^{\prime}+\lambda_{2} k_{2}$ hence $\lambda_{1} k_{1}^{*}-\lambda_{2} k_{2}^{*}=\lambda_{1}^{\prime} k_{1}^{\prime *}-\lambda_{2}^{\prime} k_{2}^{\prime *}$. Let |||||| be a strictly convex norm on $A^{*}(S)$ equivalent to the original one. The existence of such a norm in an $L$-space is proved in [3]. Now the norm $\left\|x^{*}\right\|^{\prime}=\left\|x^{*}\right\|+\left\|\mid T\left(x^{*}\right)\right\|$ is a strictly convex norm on $A^{*}(K)$ equivalent to the $\|$ \|-norm. But this is a contradiction since $l_{\infty}(\Gamma)=A^{*}(K)$ is not isomorphic to any strictly convex space [4].

3. The following lemma is due essentially to Namioka and Phelps [10]. We include here its proof since we need a result which is a bit different from that stated in [10].

Lemma 2. Let $X$ be a normed space and $K \subset X$ a compact convex set. If for any $\varepsilon>0$ there is a continuous affine map $\varphi$ of $K$ onto a finite dimensional set of $X$ such that $\|\varphi(x)-x\|<\varepsilon$ for any $x \in K$ then for any $\varepsilon>0$ there is a bounded linear map $T$ of $X$ onto a finite dimensional space of $X$ such that $\|T(x)-x\|<\varepsilon$ for all $x \in K$.

Proof. Let $\varphi: K \rightarrow X$ be a continuous affine map. One can extend $\varphi$ to a continuous affine map of $K^{\prime}=\operatorname{conv}(\{0] \cup K)$ into $X$. Indeed, if $0 \in K$ there is nothing to prove and if $0 \notin K$ but each ray emerging from the origin meets $K$ in at most one point we define $\varphi(0)=0$ and complete the definition of $\varphi$ by linearity. Finally, if $0 \notin K$ and for some $x \neq 0$ one has $\lambda x, x \in K$ with $0<\lambda<1$ then we define $\varphi(0)=$ $(1-\lambda)^{-1}(\varphi(\lambda x)-\lambda \varphi(x))$ and again we can extend $\varphi$ by linearity. It is easy but tedious to check that $\varphi$ is well defined in this manner and it is continuous and affine. If $\varphi(K)$ is finite dimensional so it is $\varphi\left(K^{\prime}\right)$. If $\|\varphi(x)-x\|<\varepsilon$ for all $x \in K$, we have the same inequality for all the points of $K^{\prime}$ or

$$
\|\varphi(x)-x\|<(1-\lambda)^{-1}(1+\lambda) \varepsilon \text { for } x \in K^{\prime},
$$

depending on how $\varphi$ was defined.

It follows from the previous remarks that there is no loss of generality in supposing $0 \in K$. From now on we follow [10]. Suppose $\varphi: K \rightarrow X$ is continuous, affine, with finite dimensional range and such that $\|\varphi(x)-x\|<\varepsilon$ for $x \in K$. Let $x_{1}, x_{2}, \cdots, x_{n}$ be a basis for the space generated by $\varphi(K)$. Then $\varphi(x)=\sum_{i=1}^{n} \varphi_{i}(x) x_{i}, \varphi_{i}$ being realvalued continuous affine functions on $K$. By [11, Proposition 4.5] there are continuous affine functionals $\psi_{1}, \psi_{2}, \cdots, \psi_{n}$ on $X$ such that $\left|\varphi_{i}(x)-\psi_{i}(x)\right|<\varepsilon\left(\sum_{i=1}^{n}\left\|x_{i}\right\|\right)^{-1}$ for $x \in K$. Thus $\left\|\varphi(x)-\sum_{i=1}^{n} \psi_{i}(x) x_{i}\right\|<\varepsilon$ so $\left\|x-\sum_{i=1}^{n} \psi_{i}(x) x_{i}\right\|<2 \varepsilon$ for $x \in K$. In particular, $\left\|\sum_{i=1}^{n} \psi_{i}(0) x_{i}\right\|<2 \varepsilon$. Defining 


$$
T(x)=\sum_{i=1}^{n}\left(\psi_{i}(x)-\psi_{i}(0)\right) x_{i}, x \in X,
$$

we have on $K$ : $\|x-T(x)\|<4 \varepsilon$. Clearly $T$ is a bounded linear map and has finite dimensional range. The proof of the lemma is complete.

Extending a little a definition given by Grothendieck [6, p. 165] we say that a pair $(X, K)$ formed by a Banach space $X$ and a compact set $K \subset X$ has the approximation property if for any $\varepsilon>0$ there is a bounded linear operator $T: X \rightarrow X$ such that $\|x-T(x)\|<\varepsilon$ for any $x \in K$ and $T(X)$ is finite dimensional.

TheOREM 3. Let $X$ be a Banach space and $S \subset X$ a compact simplex. The pair $(X, S)$ has the approximation property.

Proof. By Lemma 2 we have only to show that for any $\varepsilon>0$ there is a continuous affine map $\varphi$ of $S$ into $X$ with finite dimensional range such that $\|\varphi(x)-x\|<\varepsilon$ for any $x \in X$. Since $S$ is separable we may suppose that $X$ is separable too (if not, we consider the closed subspace of $X$ generated by $S$ ). Then by [1] there is a bounded linear map $T$ of $l_{1}$ onto $X$ with $\|T\|=1$. The multivalued map $T^{-1}$ from $S$ to the family of subsets of $l_{1}$ given by $T^{-1}(x)=\left\{y \in l_{1}: T(y)=x\right\}$ is lower semicontinuous and affine in the sense of [8]. Moreover, $T^{-1}(x)$ is a nonvoid closed subset of $l_{1}$ for each $x \in S$. According to [8, Th. 3.1] $T^{-1}$ admits a continuous affine selection, i.e., there is a continuous affine function $u: S \rightarrow l_{1}$ such that $T(u(x))=x$ for each $x \in S$. Now, the pair $\left(l_{1}, u(S)\right)$ has the approximation property since $l_{1}$ has a basis. Let $v=u(S) \rightarrow l_{1}$ be a continuous affine map with finite dimensional range such that $\|v(y)-y\|<\varepsilon$ for each $y \in u(S)$. Let $\varphi$ be the composite map $T \circ v \circ u$. Clearly $\varphi$ is continuous, affine, maps $S$ into $X$ and $\varphi(S)$ is finite dimensional. For any $x \in S$ we have

$$
\|\varphi(x)-x\|=\|\varphi(x)-T(u(x))\| \leqq\|v(u(x))-u(x)\|<\varepsilon
$$

and this completes the proof of the theorem.

4. Lemma 4. Let $(\mathscr{R}, \Sigma, \mu)$ be a measure space and $\left\{g_{n}\right\}_{n=0}^{\infty} a$ sequence in $L_{1}(\mathscr{R}, \Sigma, \mu)$. Suppose that there is a constant $M$ such that $g_{0} \geqq 0, g_{0}+\sum_{n \in N} g_{n} \geqq 0$ and

$$
\int_{\Omega^{2}} g_{0} d \mu+\sum_{n \in N} \int_{\Omega} g_{n} d \mu \leqq M
$$

for any finite set $N$ of positive integers. Then $\sum_{n=0}^{\infty} \int_{\mathscr{\Omega}}\left|g_{n}\right| d \mu<\infty$. Proof. Since 


$$
\left\|\sum_{n \in N} g_{n}\right\| \leqq\left\|g_{0}+\sum_{n \in N} g_{n}\right\|+\left\|g_{0}\right\| \leqq M+\left\|g_{0}\right\|
$$

for any finite set $N$ of natural numbers, it follows from [4, p. 60] and the weak completeness of $L_{1}(\mathscr{R}, \Sigma, \mu)$ that the series $\sum_{n=0}^{\infty} g_{n}$ is weakly (unconditionally) convergent. Denote $g=\sum_{n=0}^{\infty} g_{n}$. For $m$ an arbitrary natural number let $\left\{E_{i}\right\}_{i=1}^{p(m)}$ be a partition of $\mathscr{R}$ in disjoint measurable sets such that each $g_{n}, 1 \leqq n \leqq m$ has constant sign on $E_{i}, 1 \leqq i \leqq p(m)$. For a fixed $i$ denote:

$$
\begin{aligned}
P_{i} & =\left\{n: g_{n} \mid E_{i} \geqq 0,1 \leqq n \leqq m\right\}, \\
Q_{i} & =\{1,2, \cdots, m\}-P_{i} .
\end{aligned}
$$

We have

$$
\sum_{n \in P_{i}} \int_{E_{i}}\left|g_{n}\right| d \mu=\int_{E_{i}}\left(\sum_{n \in P_{i}} g_{n}\right) d \mu \leqq \int_{E_{i}} g d \mu
$$

and

$$
\sum_{n \in Q_{i}} \int_{E_{i}}\left|g_{n}\right| d \mu=-\int_{E_{i}}\left(\sum_{n \in Q_{i}} g_{n}\right) d \mu \leqq \int_{E_{i}} g_{0} d \mu
$$

Hence

$$
\sum_{n=1}^{m} \int_{E_{i}}\left|g_{n}\right| d \mu \leqq \int_{E_{i}}\left(g_{0}+g\right) d \mu
$$

and from this we infer

$$
\sum_{n=1}^{m} \int_{\Omega}\left|g_{n}\right| d \mu \leqq \int_{\Omega}\left(g_{0}+g\right) d \mu .
$$

The next theorem gives a necessary condition for a bounded subset of a locally convex space to be contained in a bounded simplex of the space.

THEOREM 5. Let $S$ be a bounded simplex of a locally convex space $E$. If $\left\{x_{n}\right\}_{n=0}^{\infty}$ is a sequence in $S$ such that $x_{0}+\sum_{n \in N}\left(x_{n}-x_{0}\right) \in S$ for any set $N$ of positive integers then $\sum_{n=1}^{\infty}\left(x_{n}-x_{0}\right)$ converges absolutely.

Proof. Consider in $E \times R$ the cone $C$ generated by $S \times 1=$ $\{(x, 1): x \in S\}$. The space $F=C-C$ is a linear lattice with the order induced by $C$. The Minkowski functional corresponding to the absolute convex hull of $S \times 1$ is a norm in $F$. It is readily seen that the order and this norm of $F$ are related by the following two properties: (1) if $x \wedge y=0$ then $\|x+y\|=\|x-y\|$; (2) if $x \geqq 0, y \geqq 0$ then $\|x+y\|=\|x\|+\|y\|$. It follows from [4, pp. 98-100] that the com- 
pletion of the normed lattice $F$ is an $L$-space (the order being that determined by the closure of $C$ ).

Let $T$ be the restriction to $F$ of the natural projection from $E \times R$ onto $E$. From the boundedness of $S$ we infer that $T$ is a continuous linear mapping from the normed space $F$ into the locally convex space $E$. Put $y_{n}=\left(x_{n}, 1\right), n=0,1,2, \ldots$. Clearly $y_{0} \in S \times 1$, $y_{0}+\sum_{n \in N}\left(y_{n}-y_{0}\right) \in S \times 1$ for any set $N$ of natural numbers. Now $S \times 1$ is part of the positive face of the unit ball in an $L$-space. By Lemma 4 we have $\sum_{n=1}^{\infty}\left\|y_{n}-y_{0}\right\|<\infty$. Since $T\left(y_{n}\right)=x_{n}$ it results from this and the continuity of $T$ that $\sum_{n=1}^{\infty}\left(x_{n}-x_{0}\right)$ is absolutely convergent.

CoRollary 6. Let $E$ be a metrizable locally convex space such that each totally bounded subset of $E$ is contained in a bounded simplex of $E$. Then $E$ is a nuclear space. In particular, in every infinite dimensional Banach space there is a compact set which is contained in no bounded simplex of the space.

Proof. By [13, p. 75] the second statement of the corollary is a consequence of the first. Let $\sum_{n=1}^{\infty} x_{n}$ be an unconditionally Cauchy series in $E$. It is easy to see that the set

$$
K=\left\{\sum_{n \in N} x_{n}: N \text { a set of positive integers }\right\} \cup\{0\}
$$

is totally bounded. Since by the hypothesis there is a bounded simplex in $E$ which contains $K$ it follows from Theorem 5 that $\sum_{n=1}^{\infty} x_{n}$ is an absolutely convergent series. Thus in $E$ every unconditionally Cauchy series converges absolutely. By [13, p. 66] $E$ is a nuclear space.

REMARK. Using the Dvoretzky-Rogers theorem [5] one can prove a stronger version of the second part of the corollary: in every infinite dimensional Banach space $X$ there is a compact subset $K$ such that in any Banach space $Z, Z \supset X$, there is no bounded simplex containing $K$.

Finally, we would like to mention some open problems:

1. Is every compact subset of a (metrizable) nuclear space contained in a compact simplex of the space?

2. Suppose that a compact (or bounded) subset $K$ of a locally convex space $E$ has the following property: if $x_{0} \in X$ and

$$
x_{0}+\sum_{n \in N^{*}}\left(x_{n}-x_{0}\right) \in K
$$

for each finite set of natural numbers then $\sum_{n=1}^{\infty}\left(x_{n}-x_{0}\right)$ converges absolutely. Is $K$ contained in a compact (respectively, bounded) 


\section{simplex of $E$ ?}

3. How can be characterized those compact subsets of the common Banach spaces which are contained in a compact simplex of the space?

\section{REFERENCES}

1. S. Banach and S. Mazur, Zur Theorie der linearen Dimension, Studia Math. 4 (1933), 100-112.

2. M.M. Day, Strict convexity and smoothness, Trans. Amer. Math. Soc. 78 (1955), $516-528$.

3. - Every L-space is isomorphic to a strictly convex space, Proc. Amer. Math. Soc. 8 (1957), 415-417.

4. - Normed linear spaces, Springer-Verlag, Berlin 1962.

5. A. Dvoretzky and C.A. Rogers, Absolute and unconditional convergence in normed linear spaces, Proc. Nat. Acad. Sci. U.S.A. 36 (1950), 192-197.

6. A. Grothendieck, Produits tensoriels topologiques et espaces nucléaires, Mem. Amer. Math. Soc. 16 (1955).

7. J. Kelley, I. Namioka and co-authors, Linear topological spaces, Van Nostrand, Princeton 1963.

8. A.J. Lazar, Spaces of affine continuous functions on simplexes, Trans. Amer. Math. Soc. 134 (1968), 503-525.

9. J. Lindenstrauss, Extension of compact operators, Mem. Amer. Math. Soc. 48 (1964).

10. I. Namioka and R.R. Phelps, Tensor products of compact convex sets, Pacific J. Math. 31 (1969), 469-480.

11. R.R. Phelps, Lectures on Choquet's theorem, Van Nostrand, Princeton, 1966.

12. - Infinite dimensional compact convex polytopes (to appear in Math. Scand.)

13. A. Pietsch, Nukleare Localkonvexe Räume, Akademie Verlag, Berlin, 1965.

14. Z. Semadeni, Free compact convex sets, Bull Acad. Polon. Sci. Sér. Sci. Math. Astronom. Phys. 13 (1964), 141-146.

Received July 2, 1969, and in revised form November 10, 1969.

UNIVERSITY OF WASHINGTON

Seattle, Washington 


\section{PACIFIC JOURNAL OF MATHEMATICS}

\section{EDITORS}

H. SAMelson

Stanford University

Stanford, California 94305

\section{Richard PIERCE}

University of Washington

Seattle, Washington 98105
J. DugundjI

Department of Mathematics

University of Southern California

Los Angeles, California 90007

BASIL GoRDON*

University of California

Los Angeles, California 90024

\section{ASSOCIATE EDITORS}
E. F. BECKENBACH
B. H. NeumanN
F. WOLE
K. YosHIDA

\section{SUPPORTING INSTITUTIONS}

UNIVERSITY OF BRITISH COLUMBIA

CALIFORNIA INSTITUTE OF TECHNOLOGY

UNIVERSITY OF CALIFORNIA

MONTANA STATE UNIVERSITY

UNIVERSITY OF NEVADA

NEW MEXICO STATE UNIVERSITY

OREGON STATE UNIVERSITY

UNIVERSITY OF OREGON

OSAKA UNIVERSITY

UNIVERSITY OF SOUTHERN CALIFORNIA

\author{
STANFORD UNIVERSITY \\ UNIVERSITY OF TOKYO \\ UNIVERSITY OF UTAH \\ WASHINGTON STATE UNIVERSITY \\ UNIVERSITY OF WASHINGTON \\ * * * * \\ AMERICAN MATHEMATICAL SOCIETY \\ CHEVRON RESEARCH CORPORATION \\ TRW SYSTEMS \\ NAVAL WEAPONS CENTER
}

The Supporting Institutions listed above contribute to the cost of publication of this Journal, but they are not owners or publishers and have no responsibility for its content or policies.

Mathematical papers intended for publication in the Pacific Journal of Mathematics should be in typed form or offset-reproduced, (not dittoed), double spaced with large margins. Underline Greek letters in red, German in green, and script in blue. The first paragraph or two must be capable of being used separately as a synopsis of the entire paper. The editorial "we" must not be used in the synopsis, and items of the bibliography should not be cited there unless absolutely necessary, in which case they must be identified by author and Journal, rather than by item number. Manuscripts, in duplicate if possible, may be sent to any one of the four editors. Please classify according to the scheme of Math. Rev. 36, 1539-1546. All other communications to the editors should be addressed to the managing editor, Richard Arens, University of California, Los Angeles, California, 90024.

50 reprints are provided free for each article; additional copies may be obtained at cost in multiples of 50 .

The Pacific Journal of Mathematics is published monthly. Effective with Volume 16 the price per volume (3 numbers) is $\$ 8.00$; single issues, $\$ 3.00$. Special price for current issues to individual faculty members of supporting institutions and to individual members of the American Mathematical Society: $\$ 4.00$ per volume; single issues $\$ 1.50$. Back numbers are available.

Subscriptions, orders for back numbers, and changes of address should be sent to Pacific Journal of Mathematics, 103 Highland Boulevard, Berkeley, California, 94708.

PUBLISHED BY PACIFIC JOURNAL OF MATHEMATICS, A NON-PROFIT CORPORATION

Printed at Kokusai Bunken Insatsusha (International Academic Printing Co., Ltd.), 7-17, Fujimi 2-chome, Chiyoda-ku, Tokyo, Japan.

* Acting Managing Editor. 


\section{Pacific Journal of Mathematics}

\section{Vol. 33, No. $2 \quad$ April, 1970}

Raymond Balbes and Alfred Horn, Projective distributive lattices ....... 273

John Findley Berglund, On extending almost periodic functions ......... 281

Günter Krause, Admissible modules and a characterization of reduced left

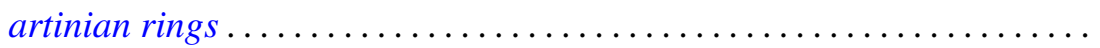

Edward Milton Landesman and Alan Cecil Lazer, Linear eigenvalues and a nonlinear boundary value problem ....................... 311

Anthony To-Ming Lau, Extremely amenable algebras ............... 329

Aldo Joram Lazar, Sections and subsets of simplexes .............. 337

Vincent Mancuso, Mesocompactness and related properties ............. 345

Edwin Leroy Marsden, Jr., The commutator and solvability in a generalized orthomodular lattice .................................. 357

Shozo Matsuura, Bergman kernel functions and the three types of canonical domains.......................................... 363

S. Mukhoti, Theorems on Cesàro summability of series .............. 385

Ngô Van Quê, Classes de Chern et théorème de Gauss-Bonnet ........... 393

Ralph Tyrrell Rockafellar, Generalized Hamiltonian equations for convex problems of Lagrange ................................ 411

Ken iti Sato, On dispersive operators in Banach lattices ............. 429

Charles Andrew Swanson, Comparison theorems for elliptic differential

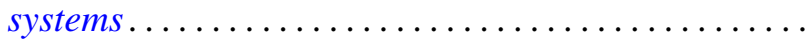

John Griggs Thompson, Nonsolvable finite groups all of whose local subgroups are solvable. II

David J. Winter, Cartan subalgebras of a Lie algebra and its ideals ... 